Research Article

\title{
Design of Nozzle and Fin Locking Unit for MRECM Rocket: A Study
}

\author{
Arjun Ogale*, Omkar Marathe, Saurabh Misal and Nitinkumar Anekar \\ Department of Mechanical Engineering, MITCOE Pune, Savitribai Phule Pune University, Maharashtra, India
}

Accepted 02 March 2016, Available online 15 March 2016, Special Issue-4 (March 2016)

\begin{abstract}
In view of the current strategic defense scenario of the world, specifically Asia, it is necessary to use modern security systems in air, land and water. One of the solutions is to have air security blanket or dome in the form of decoy missiles to intercept incoming enemy missiles. The current work was undertaken after the testing and analysis of the multi-nozzle stabilizer unit. A design for a single nozzle unit for the same rocket is now required. This paper covers the first half of a year-long effort which involves conceptualization and the initial design of the nozzle and stabilizing mechanism.
\end{abstract}

Keywords: Lift and drag, convergent-divergent nozzle, compressible flow, Mach number, weight reduction

\section{Introduction}

A counter-measure rocket is a decoy rocket that is deployed when an incoming rocket is detected in order to aggressively intercept it mid-flight to protect the attacking rocket's original target. In order for two rockets to collide mid-flight, it becomes necessary to ensure that the flight trajectory of the counter-measure rocket does not deviate from what is intended. Hence the need for a robust and precise stabilizer unit. The analysis and the results obtained for the stabilizer unit for the Medium Range Electronic Counter Measure (MRECM) rocket can be used for similar rocket systems with a few modifications in the design for launching them from various platforms. The incorporation of a single nozzle in place of the six nozzles would significantly reduce the weight of the stabilizer unit as well as reduce the complexity of the stabilizer unit. T. Manivannan describe the design, analysis and development of fin locking mechanism for fin deployment system. The design is based on a general four-bar mechanism. Mukkane bring out design of plan from configuration of wrap-around fins of a stabilization mechanism, theoretical approach for estimation of cant angle and spin. F.M.White describes in detail the theory of compressible fluid flow. Thrust forces and exhaust gas parameters have been defined by Sutton.

\section{Forces on a rocket}

In flight, a rocket is subjected to four forces; weight, thrust, and the Aerodynamic forces, lift and drag. The magnitude of the weight depends on the mass of all of

*Corresponding author: Arjun Ogale the parts of the rocket. The weight force is always directed towards the center of the earth and acts through the center of gravity, the yellow dot on the figure. The magnitude of the thrust depends on the mass flow rate through the engine and the velocity and pressure at the exit of the nozzle. The thrust force normally acts along the longitudinal axis of the rocket and therefore acts through the center of gravity. The magnitude of the Aerodynamic forces depends on the shape, size, and velocity of the rocket and on properties of the atmosphere. These forces act through the center of pressure. During the flight of a rocket small gusts of wind, or thrust instabilities can cause the rocket to "wobble", or change its attitude in flight. Like any object in flight, a rocket rotates about its center of gravity.

The rotation causes the axis of the rocket to be inclined at some angle a to the flight path. Whenever the rocket is inclined to the flight path, a lift force is generated by the rocket body and fins, while the Aerodynamic drag remains fairly constant for small inclinations. Lift and drag both act through the center of pressure of the rocket. The lift and the drag forces move the nose back towards the flight direction only if the center of pressure is located below the center of gravity. In this case, they are called 'restoring' forces. On a contrary, if the center of pressure is above the center of gravity, the lift and drag forces maintain their directions but the direction of the torque generated by the forces is reversed. This is called a de-stabilizing force. Any small displacement of the nose generates forces that cause the displacement to increase. Thus, one of the main conditions for a stable rocket is that the center of pressure must be located below the center of gravity. This principle is used while 
determining fin area and weight of stabilizer unit. The following line diagram indicates the different forces on a rocket in flight.

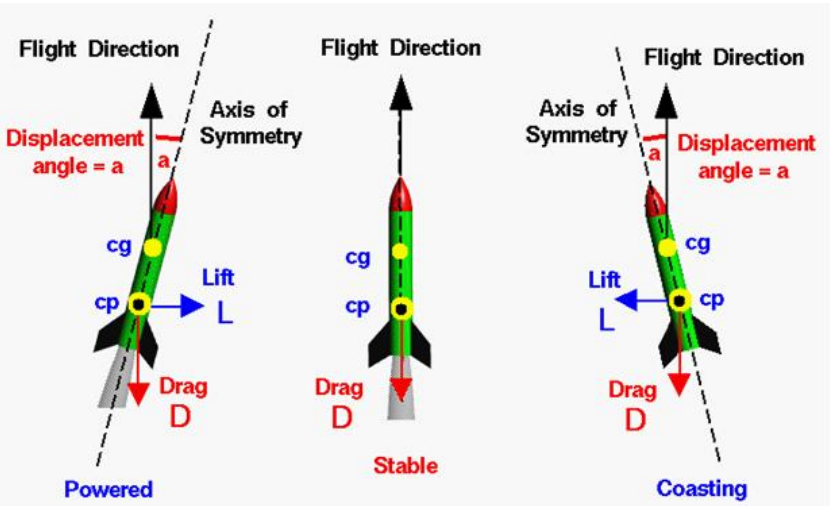

Fig. 1 Forces on a rocket

\section{Design of Stabilizer Unit and Nozzle}

\subsection{Initial design of stabilizer unit}

The initial design of the stabilizer unit consisted of one central nozzle surrounded by six peripheral nozzle, six fins and a locking ring. All these parts were contained within the nozzle block whose outer diameter was the same as the diameter of the rocket body. The function of the locking ring is to ensure that all the six fins are deployed simultaneously as the rocket leaves the launching tube. The following diagram shows the assembly of the old stabilizer unit.

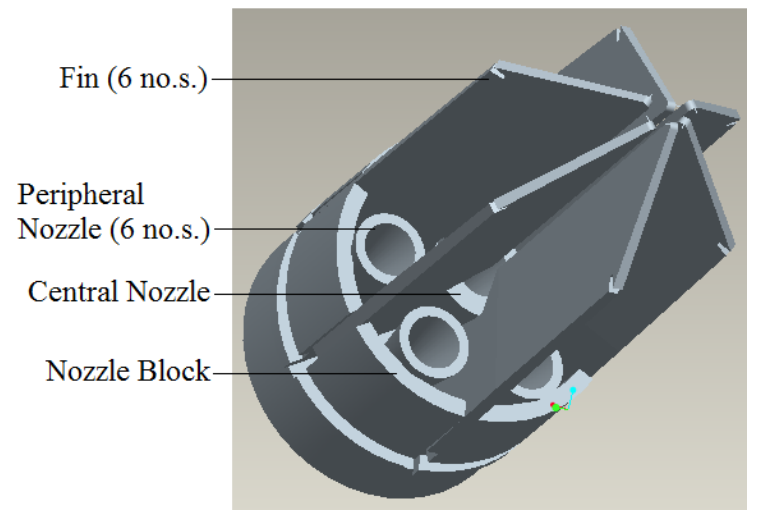

Fig. 2 Initial design of stabilizer unit

It also functions to ensure that the fins stay deployed at an angle of $45^{\circ}$ once they are opened. During deployment, the locking ring is pushed outward via a piston by the force generated by the burning of the propellant.

\subsection{New design of the stabilizer unit}

The new design of the stabilizer unit for the MRECM Rocket aims to replace the seven-nozzle system with a single central nozzle system. It consists of a nozzle block, six fins, one locking ring, one central nozzle, one compression spring and three deployment pistons. The pistons serve as an alternate deployment arrangement in case the spring fails. Typically, rockets are kept in storage for extended periods of time. It is due to this that the compression spring used under the locking ring might jam, causing the deployment to fail. The alternate deployment mechanism that uses pistons is activated by the force generated by the burning propellant. This combined compression-spring-cumdeployment-pistons system ensures a fail-safe way to deploy the six stabilizing fins. The purpose of using a single central nozzle is to reduce the weight and complexity of the stability unit. The following diagram shows the assembly of the new stabilizer unit.
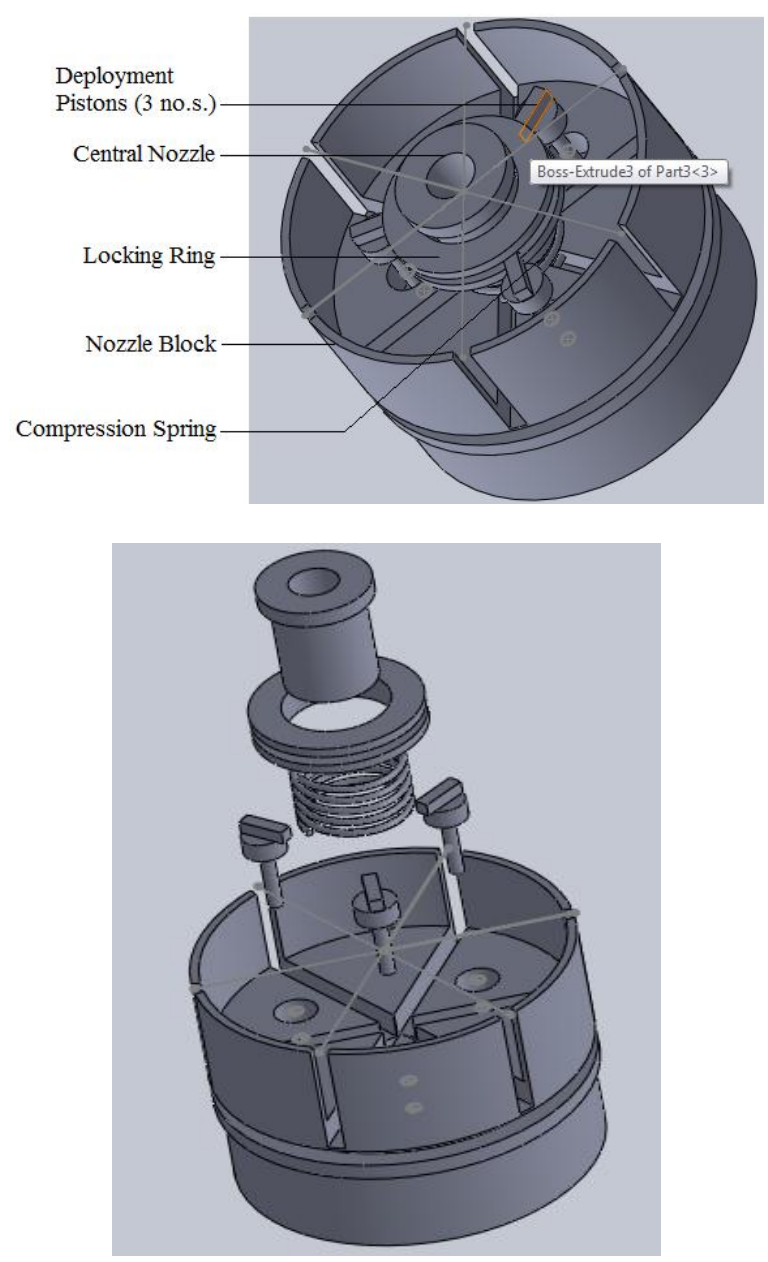

Fig. 3 New design of stabilizer unit

\subsection{Propulsion system design}

The impulse required by the rocket needs to be found out initially based on parameters like range of the rocket, mass of the payload to be carried, drag coefficient etc. CFD analysis would prove very helpful in finding out the above parameters.

The impulse is given as

$$
I=T \times t
$$


The required impulse can be achieved either by higher thrust for short duration or lower thrust for longer duration. Usually first approach is preferred for better fight stabilization. Based on the impulse required, the type of propellant with requisite ballistic properties is chosen. The physicochemical and ballistic properties are obtained by evaluation of propellant in ballistic evaluation motor (BEM are

- Calorimetric value (Cal val)

- Characteristic velocity $\left(C^{*}\right)$

- Specific heat ratio $(k)$

- Burn rate $(r)$

- Pressure index $(n)$

- Temperature sensitivity $(\Pi p)$

- Specific impulse $\left(I_{S P}\right)$

BEM motor firing at different chamber pressures is required for finding temperature \& pressure indices. The rate of burn of propellant grain follows the following relations

$$
r=a P^{n}
$$

Once foresaid parameters are obtained the mass of propellant to be used is decided based on specific impulse value (ISP).

Mass of propellant needed to obtain required impulse will be

$m=\frac{I}{I_{S P}}$

Then the propellant grain is designed based on the chamber pressure required, dimensions of the rocket and type of thrust required. Grain design can be of three types:

a. Neutral burning type in which the surface area of burning remains constant.

b. Progressive burning type in which the surface area increases with time.

c. Digressive burning type in which the surface area decreases with time.

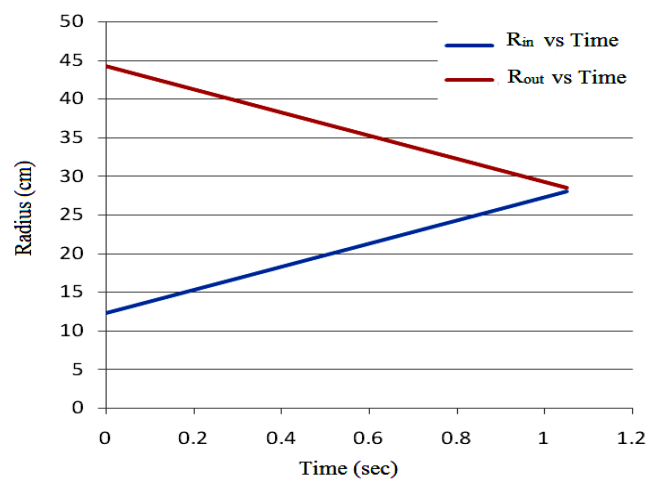

Fig. 4 Radius vs time
Based on the type of thrust required grain type is selected as thrust generated is directly proportional to mass flow rate of gases. In the mentioned rocket we used a neutral burning type grain. The geometry is hollow cylinder with inhibited ends. The graphs below give the Thrust vs time and inner and outer radius vs time relations

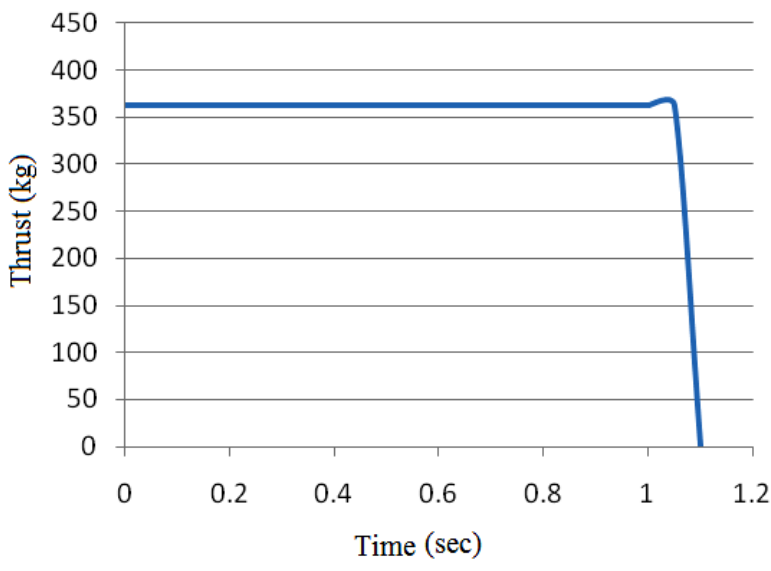

Fig. 5 Thrust vs Time

\subsection{Design of nozzle}

According to Newton's Third Law of motion, hot exhaust gases which are accelerated using a nozzle produce thrust on the rocket. The nozzle design governs the pressure at the exit of the engine, mass flow rate through the engine and the exit velocity of the flow which in turn decide the amount of thrust generated. A convergent-divergent nozzle has been used in this case. The throat size is calculated for required chamber pressure and required thrust level. The value of the Mach number at the throat is 1 i.e. the flow is sonic. The geometry diverges downstream and the flow expands isentropically to a supersonic value of the Mach number which causes the static temperature and pressure to decrease from the throat to the exit Thus, the exit temperature and pressure depend on the amount of expansion. The exit temperature determines the exit speed of sound, which in turn determines the exit velocity. The exit pressure, velocity, and mass flow through the nozzle determine the amount of thrust produced by the nozzle.

Once the chamber pressure is known the throat area of the nozzle is found out using the equation:

$$
A_{t}=\left(\frac{S \times \rho \times a \times c^{*}}{g}\right) \times P_{o}^{(1-n)}
$$

Once the throat dimensions are known, following equations are used for governing the local parameters in a nozzle: 
For finding out local temperature is given as,

$$
\frac{T_{o}}{T}=\left[1+\left(\frac{(K-1)}{2}\right) \times M^{2}\right]
$$

For finding local pressure is given as,

$$
\frac{P_{o}}{P}=\left[1+\left(\frac{(K-1)}{2}\right) \times M^{2}\right]^{\left(\frac{k}{k-1}\right)}
$$

For finding local density is given as,

$$
\frac{\rho_{o}}{\rho}=\left[1+\left(\frac{(K-1)}{2}\right) \times M^{2}\right]^{\left(\frac{1}{k-1}\right)}
$$

Velocity of sound is given as,

$$
s=(K \times R \times T)^{0.5}
$$

Area ratio is given as,

$$
\frac{A}{A_{t}}=\frac{1}{M}\left[\left\{\frac{1+\left(\frac{(K-1)}{2}\right) \times M^{2}}{1+\left(\frac{(K-1)}{2}\right)}\right\}\right]^{\left(\frac{k+1}{2 k-2}\right)}
$$

Then the pressure ratio to be maintained is decided since it controls the coefficient of thrust of the nozzle by the relation is given as,

$$
C_{f}=\left[\left(\frac{2 k^{2}}{k-1}\right) \times\left(\frac{2}{k-1}\right)^{\left(\frac{k+1}{k-1}\right)} \times\left(1-\frac{P_{e}}{P_{c}}\right)^{\frac{k-1}{k}}\right]+\left[\left(\frac{P_{e}-P_{o}}{P}\right) \times \frac{A_{e}}{A_{t}}\right]
$$

The length of the nozzle is decided by using trigonometric relations where

$$
L=\frac{R_{\text {exit }}-R_{\text {throat }}}{\tan (\text { angle of divergence })}
$$

Where angle of divergence is kept below $13^{0}$ for reducing flow losses.

If the length of the nozzle does not fit in the design the exit pressure needs to be reworked and corresponding coefficient of thrust needs to be found out.

Thrust $=C_{f} \times A_{t} \times P$
It should satisfy the given impulse which is as givn in equation (1). Through iterative processes the given impulse requirements are met and then the design is completed for the nozzle.

The following graphs are provided for variation of different parameters along the nozzle for our design:

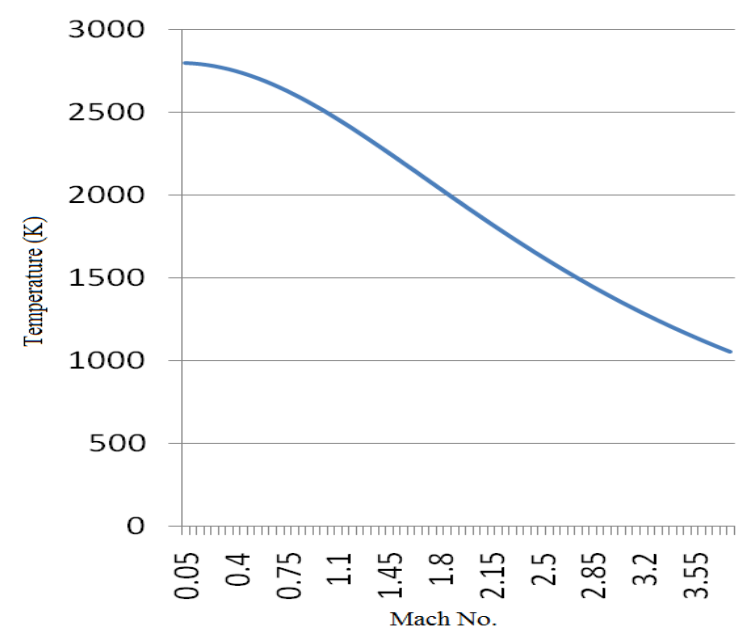

Fig. 6 Temperature vs Mach No.

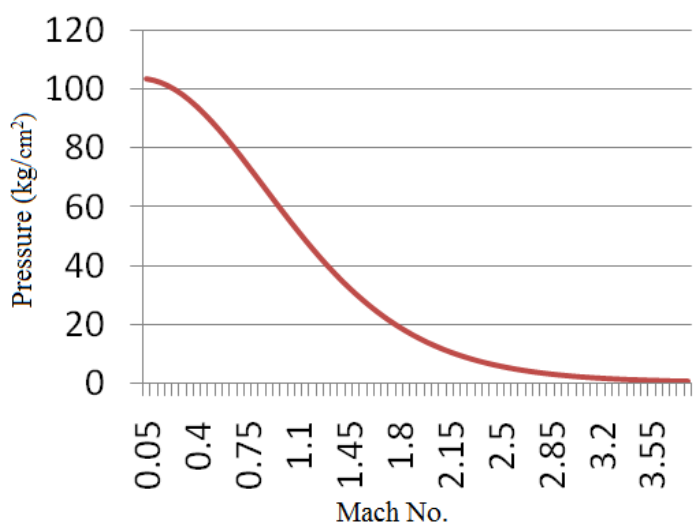

Fig.7 Pressure vs Mach No.

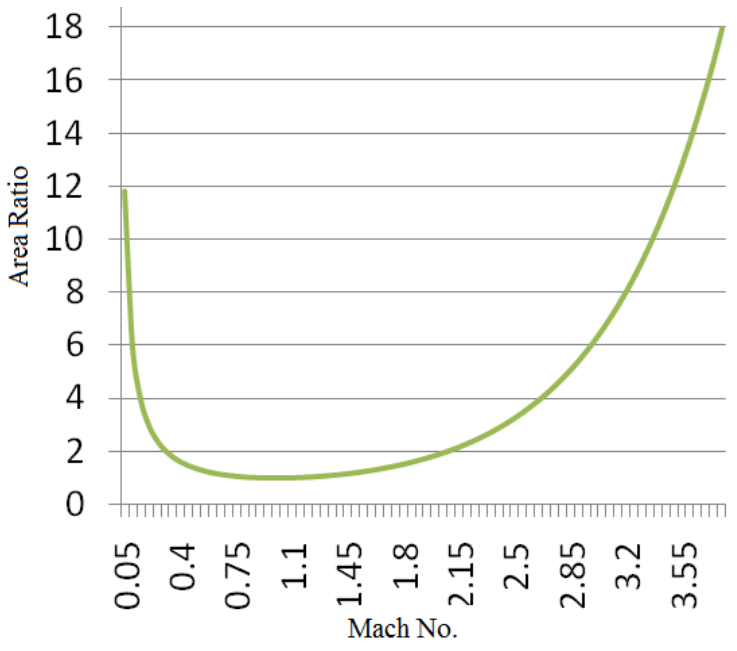

Fig. 8 Area Ratio vs Mach No. 


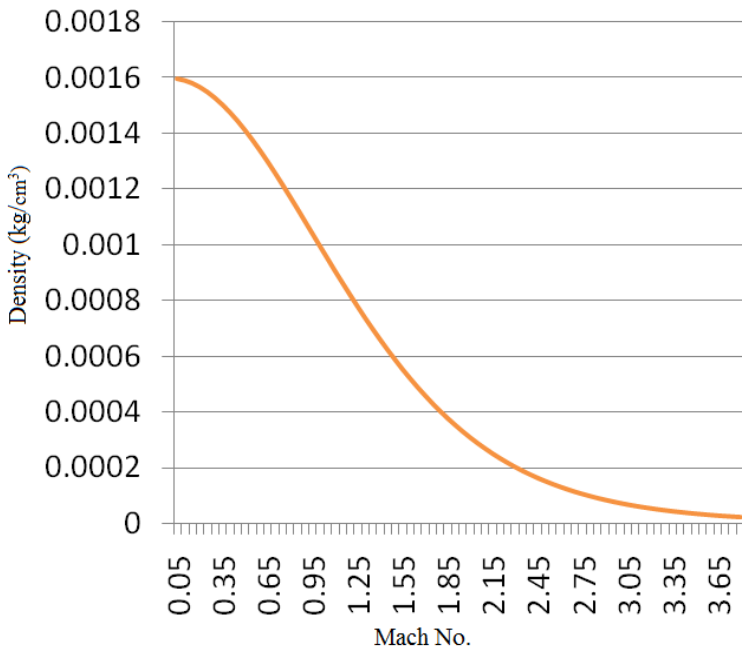

Fig. 9 Density vs Mach No.

\section{Advantages and applications of MRECMR}

The advantages over the previous stabilizer unit are given as,

- Thrust alignment with body axis.

- Reduction in complexity of the stabilizer unit.

- Reduction in manufacturing cost

- Reduction in skin friction losses.

Applications of this Rocket are given as,

The need for this project arose in the naval forces after electronics were started to be used in guiding offensive missiles to their targets.

\section{Discussion}

From initial design of nozzle it can be concluded that it is feasible to replace multiple nozzle propulsion system with a single nozzle propulsion system for the required impulse which helps in keeping the skin friction losses to a minimum as skin contact area reduces. The components of the initial design have been modelled using the software Pro-Engineer. The newer design was done in Solidworks software. This design will now be subjected to computational fluid dynamics analysis and field trials to check its robustness at high air speeds.

\section{Nomenclature}

$\mathrm{P}_{0} \quad$ Stagnation pressure

$\rho \quad$ Density of propellant

$\rho_{0} \quad$ Local density

V Local velocity

P Local pressure

K Specific heat ratio

M Local Mach no

$\mathrm{T}_{0} \quad$ Stagnation temperature

$\mathrm{T}$ Local temperature

A Local area

$\mathrm{A}_{\mathrm{e}} \quad$ Exit area

$s \quad$ Local speed of sound

$A_{t} \quad$ Throat area

$\mathrm{I}_{\mathrm{sp}} \quad$ Specific impulse

$\mathrm{C}_{\mathrm{f}} \quad$ Coefficient of thrust

M Mass

$\mathrm{P}_{\mathrm{t}} \quad$ Throat pressure

$\mathrm{P}_{\mathrm{e}} \quad$ Exit pressure

S Surface area

N Pressure indices

A Temperature indices

$\mathrm{K} \quad$ Specific heat ratio

C* Characteristic velocity

$\mathrm{R} \quad$ Burn rate

I Impulse

T Thrust

T Time

\section{Acknowledgements}

We are grateful for all the support given by our head of department of Mechanical Engineering and the principal of MIT College of Engineering, Pune.

\section{References}

Md. Shahid Quamar, T. Manivannan (2001), Design, Analysis and Testing of Fin Locking Mechanisms for Fin Deployment System of an Aircraft External Store, ASMD

S. B. Mukkane, K. J. Mane, S. M. Shelar, Dr. K. M. Rajan, (2013) Design and Development of an Innovative Mechanism for Deployment of WAF Stabilization System for an Artillery Rocket, ASMD

Jean-Pierrie, Bizien Rene, G. Patouilliart, Michel George, (1976) Artillery Projectile with Spreading Tail Assembly, United States Patent no. 3,944,168

F. M. White,(1999) ,Compressible flow, Fluid Mechanics, International edition, McGraw-Hill.

Sutton, Oscar Biblorz, Rocket propulsion Elements, $7^{\text {thedition, }}$ Wiley Interscience.

Jadon V. K., Verma Suresh(2010), Machine Design Data Book, I. K. International Publication House, 